\title{
Powered wheelchair simulator development: implementing combined navigation-reaching tasks with a 3D hand motion controller
}

\author{
Gordon Tao ${ }^{1,2^{*}}$ and Philippe S. Archambault ${ }^{1,2}$
}

\begin{abstract}
Background: Powered wheelchair (PW) training involving combined navigation and reaching is often limited or unfeasible. Virtual reality (VR) simulators offer a feasible alternative for rehabilitation training either at home or in a clinical setting. This study evaluated a low-cost magnetic-based hand motion controller as an interface for reaching tasks within the McGill Immersive Wheelchair (miWe) simulator.

Methods: Twelve experienced PW users performed three navigation-reaching tasks in the real world (RW) and in VR: working at a desk, using an elevator, and opening a door. The sense of presence in VR was assessed using the iGroup Presence Questionnaire (IPQ). We determined concordance of task performance in VR with that in the RW. A video task analysis was performed to analyse task behaviours.

Results: Compared to previous miWe data, IPQ scores were greater in the involvement domain $(p<0.05)$. Task analysis showed most of navigation and reaching behaviours as having moderate to excellent $(K>0.4$, Cohen's Kappa) agreement between the two environments, but greater $(p<0.05)$ risk of collisions and reaching errors in VR. VR performance demonstrated longer $(p<0.05)$ task times and more discreet movements for the elevator and desk tasks but not the door task.

Conclusions: Task performance showed poorer kinematic performance in VR than RW but similar strategies. Therefore, the reaching component represents a promising addition to the miWe training simulator, though some limitations must be addressed in future development.
\end{abstract}

Keywords: Virtual reality, Powered wheelchair, Training, Reaching, Navigation, Rehabilitation

\section{Background}

Reaching for objects, along with manoeuvrability within confined spaces, is a key factor in powered wheelchair (PW) mobility, i.e. the ability to overcome the physical and social obstacles of daily activities [1]. The ability to reach is necessary in a wide variety of common tasks: preparing food, working at an office, etc. Considering that PW users typically spend all their waking hours in their wheelchair [2, 3], learning how to best navigate their PW

\footnotetext{
* Correspondence: taogordon@gmail.com

${ }^{1}$ School of Physical and Occupational Therapy, McGill University, 3654 prom Sir-William-Osler, Montréal, QC H3G 1Y5, Canada

${ }^{2}$ Interdisciplinary Research Center in Rehabilitation (CRIR), Hôpital Juif de Réadaptation, 3205 Place Alton Goldbloom, Laval, QC H7V 1R2, Canada
}

in order to reach for objects is crucial to their independence and quality of life. This advanced task-related training is often not possible in rehabilitation centers as access to training for PWs is already limited $[4,5]$.

Virtual reality (VR) simulators offer a highly feasible supplement for rehabilitation training either at home or in a clinical setting. Several simulators for PW are already in development [6-8]. Critical to the effectiveness of VR training is the transfer of skills to real-world scenarios [9-11]. To simulate reaching tasks for training, motion capture technology may be used to recreate the user's manual movements in the VR environment, in real-time. In VR research, however, this usually requires large and expensive 3D cameras that are impractical for 
an at-home simulator [12-14]. Recent advancements in consumer-level motion-capture technology provide a low-cost and portable substitute. The focus of this study was to validate a six degree-of-freedom hand motion controller (Razer Hydra, Sixense, USA), as a training tool for reaching tasks in PW use. The Razer Hydra is a device that fits into one hand, similar in shape to a TV remote. The motion capture aspect of this device allows the user to physically control a virtual hand or cursor in 3D space with hand and arm movements. We chose this device for its low cost (\$150 USD), portability, and ease of use (plug \& play USB connection). This 3D hand motion controller was evaluated as an integrated tool in the miWe simulator.

An important consideration in VR research is the user's sense of presence (SOP), i.e. the temporary suspension of disbelief such that users feel as if they were 'in' the VR environment [11]. Accordingly, presence is thought to make tasks in the VR environment feel more natural and relevant to the user and therefore could enhance task training and transfer of task performance to the real world (RW) environment. The SOP can be enhanced through visual immersion in the virtual world via head mounted display or large projection screens [8]. However, a greater degree of interactivity or the number of things that can be authentically performed in the virtual environment can also contribute to a greater SOP $[15,16]$. Therefore, the addition of a 3D hand motion controller is expected to contribute to a greater SOP by providing a means of interacting with the VR environment using one's hands.

The Hydra motion controller was integrated into the McGill Immersive Wheelchair (miWe) simulator, a VR training simulator for PW users. The miWe is a firstperson perspective environment that runs on an ordinary computer. The virtual PW is controlled using a common PW joystick modified to connect to the simulator via USB. It can be used at home and is designed to teach PW users navigation and obstacle avoidance skills in the outside community. Manual tasks, such as opening doors, in the miWe were originally accomplished by keystroke. This project enabled users to physically perform such manual tasks in combination with manoeuvring their wheelchair in the simulator.

The objectives of this study were to:

1) determine to what extent using a $3 D$ hand motion controller with the miWe simulator increases the SOP of PW users compared to those who use the simulator without the hand motion controller, and

2) compare, in a group of PW users, the performance of combined navigation-reaching tasks in a PW simulator using, using the hand motion controller, to those same activities performed in real life,
3) expound the way in which participants feel the combined navigation-reaching tasks, in context of the simulator, are appropriate for PW training.

\section{Methods}

This study was approved by the Institutional review board of the Interdisciplinary Research Center in Rehabilitation (CRIR), reference number CRIR-728-0412.

\section{Population and sample}

This study focused on the participation of experienced PW users, with a minimum of 2 years' experience, as they were most likely to have stable behaviour and know how best to perform tasks; they were presumed to be familiar with scenarios encountered during activities of daily living. These users could provide expert opinions and feedback on the overall learning utility of the added reaching feature to the simulator from a standpoint of retrospective experience.

Participants were recruited from the Lucie Bruneau Rehabilitation Center (Canada). Participants needed to have a standard indoor/outdoor PW controlled by joystick. Other inclusion criteria consisted of: able to fully understand the tasks with a score of 13 or more on the Montreal Cognitive Assessment [17], have one able arm and hand for controlling the wheelchair, able to grasp at $40 \mathrm{~cm}$ forward and laterally, and normal or corrected vision. Potential participants were asked if they were able to perform the three tasks without assistance and only those who affirmed they could were recruited. Participants were excluded if they were unable to answer questions in French or English.

\section{Ethics, consent and permission}

Participants provided informed consent to participate according to CRIR guidelines.

\section{Consent to publish}

Participants provided informed consent to have their collected data published.

\section{Procedures}

Participants performed three tasks in the RW and in the miWe simulator (VR). These tasks were designed to reflect a variety of hand-arm movements (pushing, pulling, turning, transporting an object) with different levels of difficulty and are illustrated in Fig. 1a.

\section{Desk}

For this task, participants began in front of the desk. On the opposite side of the desk from where the participant began were two chairs with enough space in between to fit the PW. On the left side of the desk was a desktop drawer and on the right side was a lightweight circular 


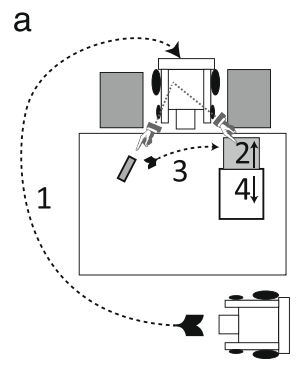

b

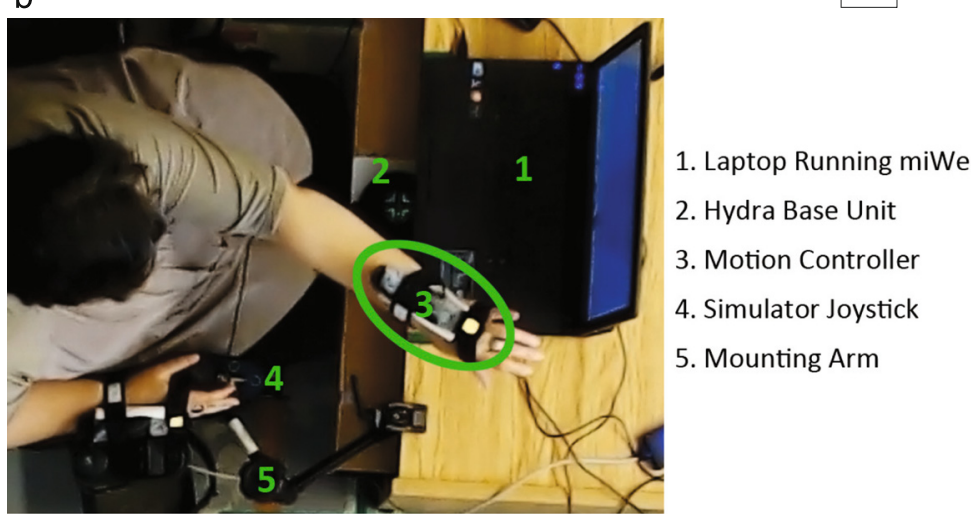

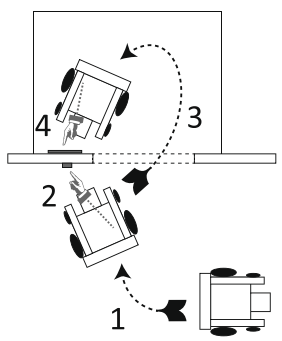

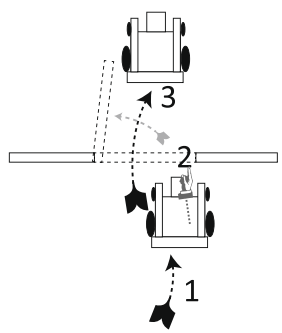

(1) 
the regular place of the PW joystick. The simulator joystick was similar to joysticks utilized by many PW models (Penny \& Giles joystick, Traxsys, UK). The Hydra motion controllers (Sixense, USA) were modified with straps to be easily attached to the back of each hand. These are capable of one-to-one position and orientation tracking in 3D space. Each controller contains a magnetic sensor that interacts with a weak magnetic field produced by a base unit, providing tracking of the controller's movement and orientation through space (accuracy of $<1 \mathrm{~mm}$ and $<1^{\circ}$ ). The advantage of this system is that it does not require line-of-sight between controller and sensor. Participants practiced navigating and interacting with objects in each virtual task (max $15 \mathrm{~min})$.

For the VR tasks, environments developed for the miWe simulator included objects (doorway, desk, etc.) that were dimensional recreations of the objects used in RW; reaching and driving movements performed by the participant were translated 1:1 into the virtual world. The virtual PW was modeled as a generalized PW based on weight, acceleration, size, and directionality. We were interested in the reaching movement only and not in hand manipulations. Therefore, manual interaction in the simulator with objects was contextual and initiated by proximity, i.e. when the participant reached close enough to the object. For example, in the door task, the virtual hand would automatically grasp the door on proximity; however, the participant needed to supinate their forearm in order to rotate the handle $45^{\circ}$, then make a pushing gesture, which would cause the door to swing completely open.

In VR, participants generally performed the Door task first, followed by the Desk then Elevator tasks. We reasoned that while participants were given time to become familiar with the simulator, some learning effects were still likely to occur. Therefore, the Door task, as the most straight-forward task and least likely to see varied strategy, was presented first. However, the ultimate order depended on the participant; sometimes a participant had difficulty completing a given task at first-this task was revisited after completing the other tasks.

\section{Data collection}

The iGroup Presence Questionnaire (IPQ) was used to determine the users' SOP while using the miWe simulator. We used the IPQ to determine if the addition of the motion-controlled reaching interaction increased SOP for the miWe. The IPQ comprises of fourteen items in four subcategories: Spatial Presence, Involvement, Experienced Realism, and a general 'sense of being there' [18]. All items are scored on a seven-point scale (0-6), with a higher score indicating a greater SOP. The IPQ has good internal consistency, with with Cronbach's alpha of 0.87 for the complete scale and approximately 0.75 for each subscore [19]. The IPQ was administered immediately after completing the all tasks in VR and RW.

For joystick position data in RW, we used a modified PW joystick connected to a data logging system [7], which sampled at $200 \mathrm{~Hz}$, that we mounted on the participant's wheelchair. We also used a ceiling-mounted wide-lens Logitech HD Pro Webcam C920 (Morges, Switzerland) with an average sampling rate of $30 \mathrm{~Hz}$ at 1080p resolution and encoded in H.264/MPEG-4 to record video data.

For VR, the miWe simulator recorded joystick input, virtual $\mathrm{PW}$ position, and motion controller position and orientation at an average sampling rate of $50 \mathrm{~Hz}$, corresponding to the simulator's video frame rate.

Participant-reported data was collected from a taskspecific and general questionnaire that prompted participants to provide feedback regarding usability, relevance, limitations, improvements, etc. for the simulator. The questionnaire was similar to the feedback form used in a previous miWe study [20]. We emphasized feedback regarding the reaching component and the motion controller. The feedback form was administered immediately after the IPQ.

\section{Analysis}

IPQ scores were compared to data gathered from a previous study on the miWe without the reaching component using an independent t-test [20]. Questionnaire data were summarized with common trends.

Video and joystick data from the RW environment were time synchronized by the manual marking of the onset of first joystick movement-the task start. Task completion time was determined by task-specific criteria: closing the drawer, pressing the floor button of the elevator, and crossing through the doorway. Furthermore, time spent reaching was defined by the onset of a reaching movement towards a reaching objective to the end of returning the arm to rest or the end of task completion criteria.

In both the RW and VR environments, joystick excursion was calculated via the vector norm of $\mathrm{x}$ and $\mathrm{y}$ displacements. Joystick movements were defined as an excursion away from the neutral position (threshold of $10 \%)$. Since the wheelchair brakes automatically engaged when the joystick was in neutral position, this definition provided a clear picture of go-stop driving patterns.

Number of reaching movements was counted by visual inspection of video data for RW tasks and from the hand position data collected from the simulator for VR (cross referenced with video data). A reaching movement was defined as 'an effortful movement towards an object'; 
thus, pressing a button counted as 2 movements (reach to press and return to rest), while reaching to grasp with lateral drifting (searching behaviour) counted as one movement.

Quantitative measures were compared across participants in pairwise fashion. Data pairs (RW, VR) were the mean of the five trials per task per environment. Wilcoxon sign-rank tests were used to compare nonnormally distributed continuous and count data and ttest was used for normally distributed data.

A video task analysis was performed to compare task errors and behaviours. For each task, two unblinded researchers identified and agreed on sub-tasks that comprised of a single goal, e.g. driving up to the door. Within each sub-task, two researchers independently identified (across all trials) on distinct behaviours that could vary from trial to trial. Any disagreement on these observations was solved by discussion until a consensus was reached. Each behaviour comprised of mutually exclusive options and both researchers agreed on strict contextual criteria for coding. One researcher coded all trials and a third blinded researcher verified the coding of 3 participants for consistency. Task behaviours included strategies (e.g. which hand was used for reaching) and performance characteristics (e.g. un/interrupted driving). Errors were evaluated similarly, but separately from task behaviours using relative risk assessment.

Concordance between RW and VR tasks was determined for each behaviour of each task. For a given behaviour, each participant's dominant behaviour in RW was identified and compared to their corresponding dominant behaviour in VR. To illustrate, we may consider the behaviour, 'drive forward or reverse into the elevator', during the Elevator task. If the participant reversed in 5/5 times in RW and 3/5 times in VR, the dominant behaviour in both environments would be 'reverse'. Therefore, behaviour, 'forward/reverse in', would be rated as concordant for the Elevator task of the participant. If, however, the participant reversed in $3 / 5$ times in RW and 2/5 times in VR, the dominant behaviour in RW would be 'reverse' and the dominant behaviour in VR would be 'forward'. Therefore, the dominant behaviour would be rated as discordant between the two environments.

Each behaviour for each participant was rated concordant/discordant (1/0); 'dominant behaviour' in this context was selected to describe the expert participants' preferred behaviour and most likely reflects their 'best practice' for task performance in RW. We wanted to see how closely VR performance matched these behaviours. Furthermore, PW users do not always perform tasks in the same manner in RW. A VR simulator that elicits authentic behaviour should also reflect this.
Concordance of task behaviours across participants was determined using Cohen's Kappa coefficient:

$$
K=\frac{\operatorname{Pr}(a)-\operatorname{Pr}(e)}{1-\operatorname{Pr}(e)}
$$

where $\operatorname{Pr}(a)$ is the proportion of counted concordant cases and $\operatorname{Pr}(e)$ is the proportion of concordant cases due to 'random chance' behaviour, e.g. the $\operatorname{Pr}(e)$ of 'used hand' would be 0.5 ; for the purposes of this study, we assumed equal proportion of all identified behaviours for $\operatorname{Pr}(\mathrm{e})$. Kappa values were evaluated according to guidelines proposed by Fleiss [21].

\section{Results}

Of fourteen people recruited, four women and eight men aged between 36 and 60 years (50.1 SD 9.1) participated in this study (Table 1). One person proved unable to perform our tasks independently on arrival and did not participate and another failed to appear due to prior injury. The participants had between 2 and 30 years (16.1 SD 9.7) experience using their PWs and an overall MOCA score of 20.1 SD 4.5. The right-handed to lefthanded ratio was 1:1.

All participants completed all tasks for both RW and VR environments. In VR, 10 of 12 participants completed the door task first. Two participants needed to complete the session over 2 days, with one environment $(\mathrm{VR} / \mathrm{RW})$ completed on each day. In both cases, the session was delayed by difficulties adapting the PW joystick logger to the participant's PW.

Measures of task completion time, reaching time, joystick movement count, and reaching movement count were averaged for each task for each participant. Distributions of these averages were found to be non-normal

Table 1 Participant descriptions

\begin{tabular}{lllllll}
\hline Participant & Age & Sex & Experience & Handed & PWC & MoCA \\
\hline 1 & 36 & M & 24 & R & Rear-Wheel & 15 \\
2 & 60 & F & 14 & L & Rear-Wheel & 19 \\
3 & 51 & F & 23 & L & Rear-Wheel & 13 \\
4 & 55 & F & 10 & L & Rear-Wheel & 25 \\
5 & 51 & M & 20 & $\mathrm{~L}$ & Mid-Wheel & 20 \\
6 & 56 & M & 30 & L & Mid-Wheel & 18 \\
7 & 56 & F & 2 & R & Rear-Wheel & 25 \\
8 & 37 & M & 25 & R & Rear-Wheel & 24 \\
9 & 59 & M & 4 & $\mathrm{R}$ & Rear-Wheel & 26 \\
10 & 59 & M & 26 & L & Rear-Wheel & 21 \\
11 & 38 & M & 10 & $R$ & Mid-Wheel & 14 \\
12 & 43 & M & 5 & $R$ & Rear-Wheel & 21 \\
\hline
\end{tabular}

Age (years), Sex (Male, Female), Experience (years), Handedness (Right, Left), MoCA $=$ Montréal Cognitive Assessment Test $(\max =30)$ 
(Shapiro-Wilk, $p<0.05)$. Therefore, the Wilcoxon signed-rank test was appropriate for comparing paired data.

\section{Presence}

Presence was measured for the VR simulator using the IPQ; scores were normally distributed and are summarized with mean and standard deviation in Fig. 2. Overall, 'general sense of presence,' 'spatial presence,' and 'realism' averaged slightly below three; only 'involvement' averaged above three. In comparison with previous data collected on the miWe [20], without a reaching component, our results showed a significantly greater (independent t-test, $p<0.05)$ sense of 'involvement'. However, scores in the remaining three categories were not significantly different.

\section{Task times}

The Elevator task took the longest total time with a median time of $24.9 \mathrm{~s}$ (interquartile range [IQR] 21.4-31.7 s) in RW and 57.6 s (IQR 42.6-69.2 s) in VR. The median time for the Desk task was $19.5 \mathrm{~s}$ (IQR 15.3-24.9 s) in RW and 52.6 s (IQR 39.4-77.4 s) in VR. The Door task took the least time overall with a median task time of $10.6 \mathrm{~s}$ (IQR 7.5-14.4 s) in RW and $13.4 \mathrm{~s}$ (IQR 42.6-69.2 s) in VR.

Only the Door task demonstrated similar task completion times with no significant difference between VR/ RW $(p=0.3)$. For the Desk and Elevator tasks, the VR completion times were both significantly $(p<0.01)$ longer; the Desk task showed the greatest difference: median $33.0 \mathrm{~s}$ (IQR 15.4-53 s) longer to complete in VR than RW. A plot of task completion time differences is shown in Fig. 3a.

Within each task in the RW, participants spent time reaching for objects a median of $6.1 \mathrm{~s}$ (IQR 4.8-9.0 s) in the Desk task, $5.2 \mathrm{~s}$ (IQR 4.1-6.7 s) in the Elevator task, and $3.8 \mathrm{~s}$ (IQR 3.4-5.6 s) in the Door task. By comparison, participants spent significantly more time

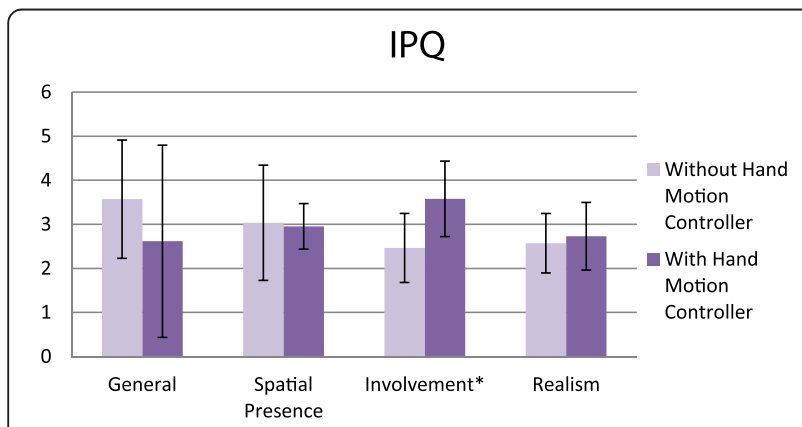

Fig. 2 iGroup Presence Questionnaire. IPQ scores are summarized across 4 domains compare previously gathered miWe data without the $3 \mathrm{D}$ hand motion controller and our results. Error bars represent standard deviation and $\left(^{*}\right)$ is significant $(p<0.05)$

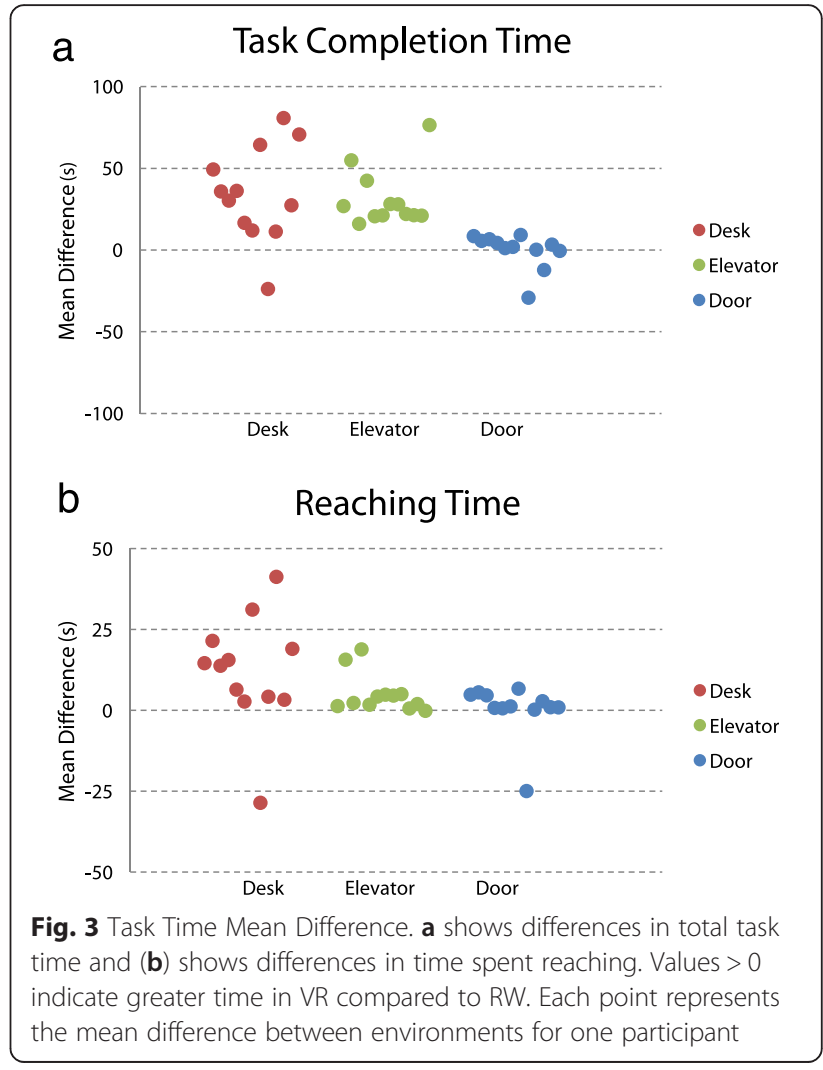

in VR for all three tasks $(p=0.02$ for Desk, $p<0.01$ for Elevator, $p=0.03$ for Door). The greatest difference in reaching time was in the Desk task where participants took a median 14.2 s (IQR 4.0-19.7 s) longer for reaching in VR than in RW. A plot of reaching time differences is shown in Fig. 3b.

\section{Discrete movements}

Figure 4a shows an example of the Elevator task performed by one participant in VR. Figure $4 \mathrm{~b}$ shows example traces representing joystick excursion during the Elevator task in RW and in VR. The complexity of the navigation component of this task is demonstrated by the number of joystick movements. Moreover, the VR trace shows noticeably more joystick movements than in the RW counterpart.

Within each task in the RW, participants completed tasks with a median number of joystick movements of 2.7 (IQR 1.8-4.5) in the Desk task, 4.4 (IQR 3.4-6.0) in the Elevator task, and 3.6 (IQR 2.4-5.2) in the Door task. By comparison, participants utilized significantly more joystick movements in VR for the Desk task $(p<$ $0.01)$ and Elevator task $(p<0.01)$. The greatest difference was in the Elevator task where the VR environment required a median of 8.4 (IQR 4.8-12.3) more joystick movements than in RW. However, joystick movement count was concordant between RW and VR in the Door 


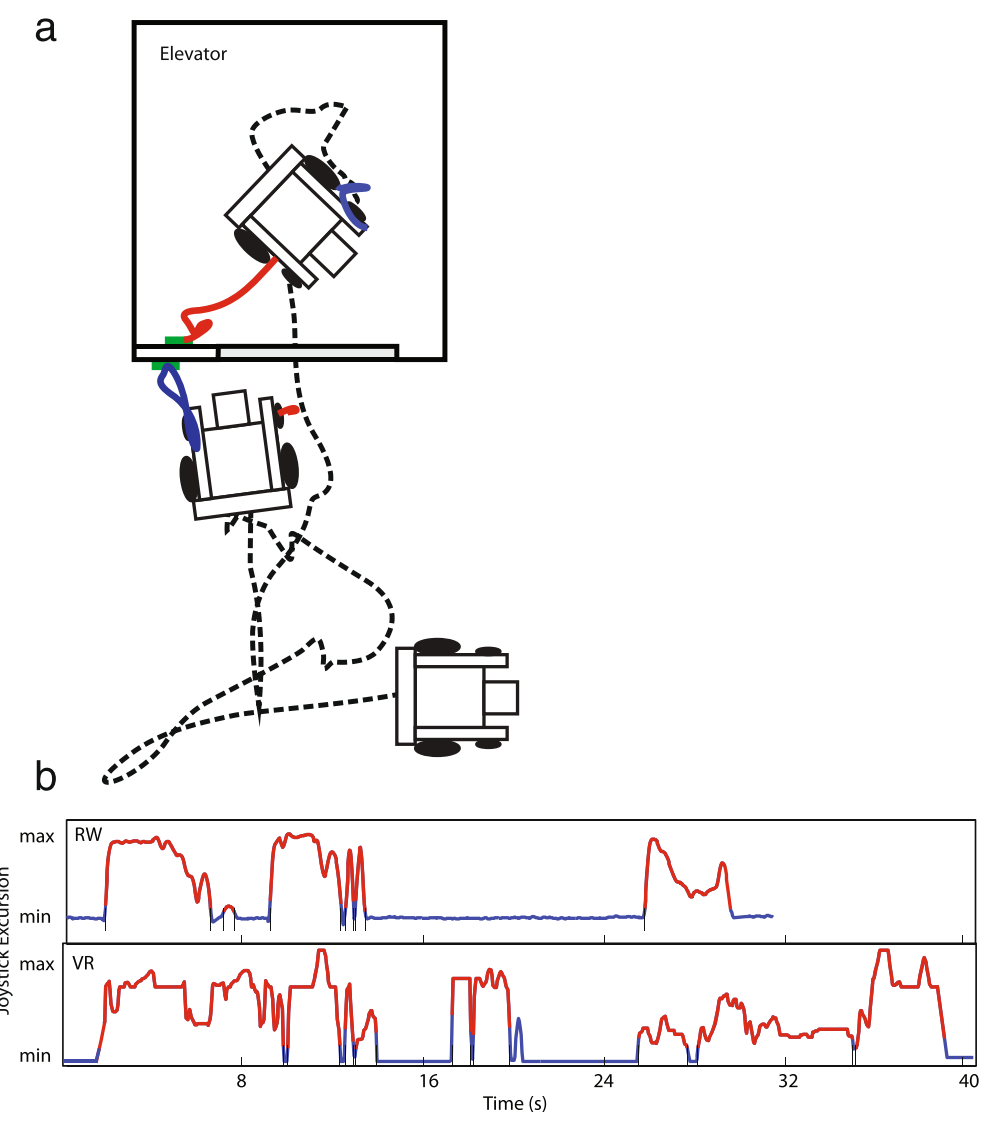

Fig. 4 Elevator Trial Examples. a show the position traces, in a VR trial, of the wheelchair (black) and hands during reaching in red and blue. $\mathbf{b}$ shows examples of joystick excursion during elevator trials in RW and VR; discrete 'joystick movements' are highlighted in red

task, with no significant difference between counts $(p=$ 0.45). A plot of joystick movement differences is shown in Fig. 5a.

Similarly, the number of reaching movements utilized was concordant between RW and VR only for the Door task $(p=0.74)$. A significantly greater number of reaching movements were made in the VR environment for the Desk task $(p<0.01)$ and Elevator task $(p=0.04)$. The greatest difference was in the Desk task where the VR environment required a median of 1.9 (IQR 1.0-2.7) more reaching movements than in RW. For comparison, participants required a median count in RW of 6.3 (IQR 6.0-6.7) in the Desk task, 3.8 (IQR 3.4-4.1) in the Elevator task, and 4.1 (IQR 3.8-4.7) in the Door task. A plot of reaching movement differences is shown in Fig. 4b.

\section{Task analysis}

Two task errors and twenty-two behaviours were identified throughout all sub-tasks. The task errors were collisions (driving error) and errors in judgment of maximum reaching distance (reaching error). A collision was defined as any contact of the PW with an obstacle.
An error in reaching distance occurred when the participant misjudged the distance required to reach a target (e.g. a button) and needed to adjust their PW position closer. Trials were divided into those where one or more driving errors occurred and those where no collisions occurred; the same procedure was done for reaching errors. For each task and across all participants, there was a significantly greater relative risk $(R R, p<0.05)$ of errors occurring during a trial performed in VR than in RW across all tasks: Desk (RR driving $=3.72$, RR reaching $=$ 2.93), Elevator (4.31, 5.86), Door (1.89, 1.5).

Table 2 describes all the sub-tasks identified and Table 3 describes all of the behaviours identified for each sub-task. These were developed from the task analysis for this study.

Performance in the Desk task showed fair $(0.4<K<$ $0.75)$ to excellent $(K>0.75)$ agreement for most reaching behaviours except for the 'close drawer hand'. However, driving behaviours showed poor agreement. Of note, the 'fluid drive-to-park' behaviour showed a negative Kappa score $(K=-0.17)$, indicating that participants tended towards the opposite behaviour in VR. Performance in the Elevator task showed generally fair agreement between 


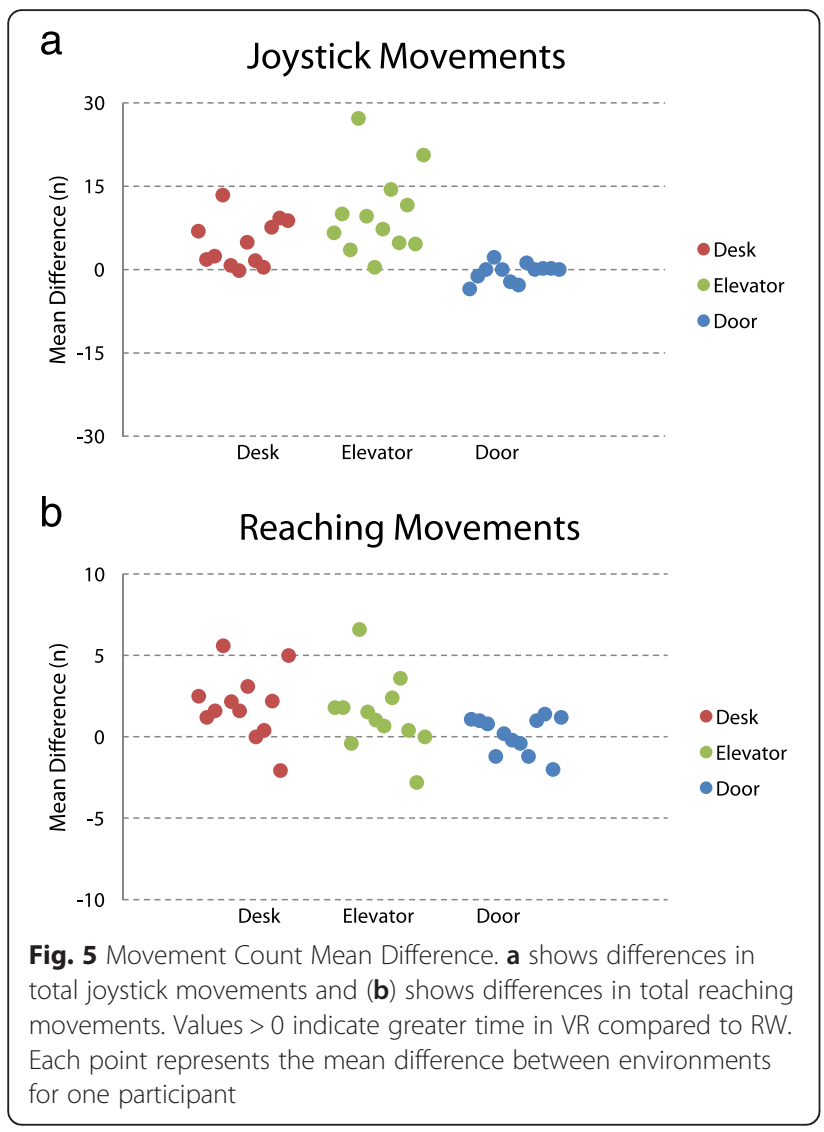

RW and VR for driving and reaching behaviours. Only the 'fluid drive-to-park' and 'waiting for door' behaviours showed poor agreement. Finally, performance in the Door task showed generally fair agreement for driving and reaching behaviours except for the 'advance straight' and 'fluid turn and push' behaviours. All agreement statistics are listed in Table 4.

\section{Questionnaire and feedback}

Participants answered additional task specific and general questions (Fig. 6) on a 5-point Likert scale (Not at all, Not very, Neutral, Somewhat, Very) and were prompted to make task specific and general suggestions and comments (Table 5) about the VR simulator.

When asked, 'Does the task seem useful for learning to use a powered wheelchair' in context of the VR simulator, 9/12 participants or more rated each of the tasks as somewhat or very useful. When asked, 'Does this task seem realistic', nearly all (10/12 or more) participants rated tasks as somewhat to very realistic. However, only $6 / 12$ to $7 / 12$ participants rated the difficulty of the simulator tasks as somewhat to very appropriate. Likewise, 5/ 12 to $6 / 12$ participants rated the tasks as somewhat to very motivating. Finally, $10 / 12$ participants rated the added realism due to the Hydra as somewhat to very much (Fig. 6b).

Participants' comments regarding specific tasks and the simulator in general varied and included both positive and negative feedback. One participant commented, "Interesting. I like the word "success" in the end, it's motivating!' Another participant commented, 'I was a little stressed. I do not like playing with joysticks in a virtual environment'. Participants were also prompted to suggest other relevant tasks; one task was suggested more than once: 'getting coffee'. A list of common comments is summarized in Table 5.

\section{Discussion}

We anticipated that the inclusion of the navigate-toreach component and the Razer Hydra 3D hand motion controller interface would increase the SOP in the miWe simulator. In the previous miWe study, where manual tasks were performed by keystroke, average scores were

Table 2 Sub-tasks. Tasks are broken down into sub-tasks and progress chronologically. Task behaviours are organized into each component. Some behaviours appear in multiple sub-tasks of the same task

\begin{tabular}{lll}
\hline Desk & Elevator & Door \\
\hline Navigating around desk & Navigate to button 1 & Advancing towards door \\
DG1, DG3, RG1 & DG1, DG3, DEl1, RG1 & DG1, DG3, DDr1, RG1 \\
Parking between chairs & Parking at button 1 & Parking in front of door \\
DG2, DG3, RG3 & DG2, DG3, RG3 & DG2, DG3, RG3 \\
Reaching for object & Pressing button 1 & Reaching for door handle \\
RG2, RDe1, RDe2 & RG2 & RG2 \\
Opening drawer & Navigate to button 2 & Opening door \\
RG2, RDe2 & DG1, DG2, DG3, DEl2, DEl3, DEl4, RG1 & RDo1 \\
Placing object in and closing drawer & Parking at button 2 & Driving through doorway \\
RDe2, RDe3 & DG2, DG3, RG3 & RDo2 \\
& Pressing button 2 & \\
\hline
\end{tabular}


Table 3 Task Behaviours. Behaviours are described in detail and given a summary code. Codes with ' $G$ ' are general behaviours observable in all tasks

\begin{tabular}{|c|c|c|c|}
\hline Code & Driving Behaviour & Code & Reaching Behaviour \\
\hline$\overline{\mathrm{DG} 1}$ & $\begin{array}{l}\text { Fluid drive-to-park: participants completed } \\
\text { navigation without pausing }\end{array}$ & RG1 & $\begin{array}{l}\text { Start reaching before park: participants began reaching before their } \\
\text { PW completely stopped }\end{array}$ \\
\hline DG2 & $\begin{array}{l}\text { Parking Position: 2-4 positions depending } \\
\text { on context }\end{array}$ & RG2 & L/R hand: which hand was used to reach the target \\
\hline DG3 & $\begin{array}{l}\text { Collision: any contact of the wheelchair with } \\
\text { an obstacle }\end{array}$ & RG3 & $\begin{array}{l}\text { Adjust parking close for reach: misjudged reaching distance, i.e. } \\
\text { reaching error }\end{array}$ \\
\hline DEl1 & $\begin{array}{l}\text { Turn first/reverse first: how participants began } \\
\text { navigating to the first button }\end{array}$ & RDe1 & $\begin{array}{l}\text { Pickup order: the object was picked up before, after, or at the same time } \\
\text { as opening the drawer }\end{array}$ \\
\hline DEl2 & $\begin{array}{l}\text { Waiting for door: participants waited for the } \\
\text { door without repositioning their PW }\end{array}$ & RDe2 & $\begin{array}{l}\text { Heavy leaning: participants needed clearly uncomfortable trunk } \\
\text { compensation to reach target }\end{array}$ \\
\hline DEl3 & Forward/reverse in: entering the elevator & RDe3 & $\begin{array}{l}\text { Close drawer hand: participants closed the drawer with either the same or } \\
\text { opposite hand that placed the object inside the drawer }\end{array}$ \\
\hline DEl4 & $\begin{array}{l}\text { Horizontal Adjust: a characteristic 'S' } \\
\text { manoeuvre sideways }\end{array}$ & RDo1 & Fluid turn + push: the door was opened in a single, non-segmented movement \\
\hline DDo1 & $\begin{array}{l}\text { Advance straight: participants drove } \\
\text { in a straight line to the door }\end{array}$ & RDo2 & Hand still raised: while driving through the doorway \\
\hline
\end{tabular}

Letter Codes: Driving (D), Reaching (R), General (G), Elevator (El), Desk (De), Door (Do)

Table 4 Task Behaviours Agreement. Summary of concordance, as measured by Cohen's Kappa coefficient (K), for each characteristic in each task across participants

\begin{tabular}{|c|c|c|}
\hline & & $\mathrm{K}$ \\
\hline \multicolumn{3}{|l|}{ Desk } \\
\hline \multirow[t]{2}{*}{ Driving Behaviour } & Fluid drive-to-park & -0.17 \\
\hline & Parking Position & 0.33 \\
\hline \multirow[t]{5}{*}{ Reaching Behaviour } & Start reaching before park & 0.83 \\
\hline & Pickup order & 0.50 \\
\hline & Close drawer hand & 0.20 \\
\hline & L/R Hand & 0.80 \\
\hline & Heavy leaning & 0.67 \\
\hline \multicolumn{3}{|l|}{ Elevator } \\
\hline \multirow[t]{6}{*}{ Driving Behaviour } & Turn First/Reverse First & 0.83 \\
\hline & Fluid drive-to-park & 0.22 \\
\hline & Parking Position & 0.45 \\
\hline & Waiting for Door & 0.17 \\
\hline & Forward/Reverse In & 0.67 \\
\hline & Horizontal adjust & 0.67 \\
\hline \multirow[t]{2}{*}{ Reaching Behaviour } & Start reaching before park & 0.67 \\
\hline & L/R Hand & 0.60 \\
\hline \multicolumn{3}{|l|}{ Door } \\
\hline \multirow[t]{3}{*}{ Driving Behaviour } & Advance straight & 0.17 \\
\hline & Fluid drive-to-park & 0.67 \\
\hline & Parking Position & 0.67 \\
\hline \multirow[t]{4}{*}{ Reaching Behaviour } & Start reaching before park & 0.50 \\
\hline & L/R Hand & 0.80 \\
\hline & Fluid turn + push & 0.33 \\
\hline & Hand still raised & 0.67 \\
\hline
\end{tabular}

above three for general sense of presence and spatial presence, but below three for involvement and realism [20]. In our study, involvement showed a significant increase in the involvement score; this likely reflects a positive effect from the added interactivity provided by the hand controllers and the increased complexity, both physically and cognitively, of combined navigationreaching tasks. Interestingly, no significant changes were observed in the other categories. We believe that drawbacks in the implementation of the virtual environment and simulation may have counteracted potential gains from the implementation of the hand controller and reaching tasks in the general, spatial presence, and realism categories of the IPQ. Examination of participant feedback, task performance, and task strategies help elucidate how this is the case.

\section{Participant feedback}

Participants responded positively with respect to training utility, realism, and graphical quality. The task difficulty and motivation were judged less positively, but were generally neutral or better. These results suggest that the overall design of the tasks and task environments were representative of tasks in RW. Participants' comments also reflected frustration with the some aspects of the simulator; they indicated that VR tasks felt more difficult to accomplish than the corresponding RW tasks, particularly because controlling the PW was harder and noticeably different from what they were used to in RW. A previous study comparing PW driving in VR vs RW showed similar reactions from participants [22]. However, several participants also commented that the reaching tasks were fun and engaging, indicating the potential for improving the motivational aspect of the simulator. 


\section{a}

'Does the task seem useful for learning to use a powered wheelchair'

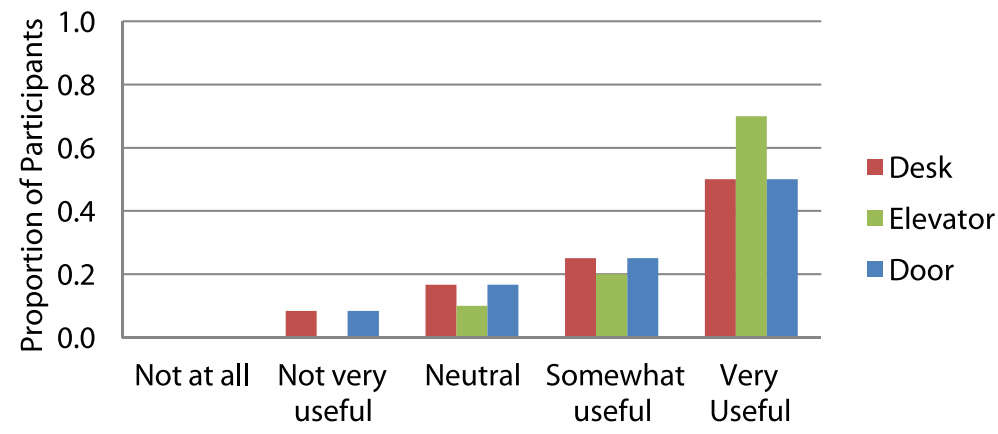

b

'Does using the hand-motion controllers enhance the

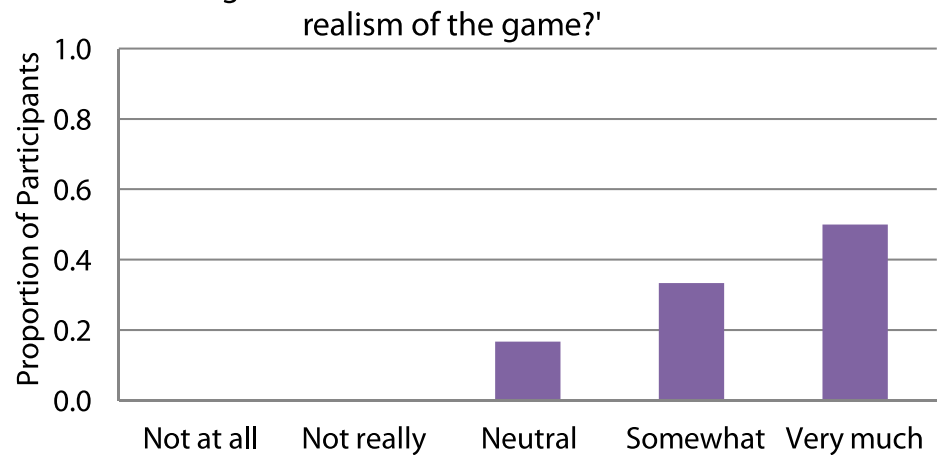

Fig. 6 Example questionnaire items, task specific (a) and general (b), and participants' responses

Overall, the feedback comments were ambivalent, with some participants responding very positively and others having severe difficulty with the simulator. One factor to consider in these responses is the novelty of the PW simulator, particularly with respect to the reaching component; since the study was cross-sectional, the novelty of the situation may have led to greater initial enthusiasm for those who already enjoyed VR experiences (e.g. videogames) and insufficient familiarization for those who were already anxious about VR environments. Furthermore, these data were from PW users recruited from one site in Montréal, which may limit generalizability. On the other hand, participants represented ages spanning a range of 23 years and experience ranging from 2 to 30 years; they were also providing feedback with respect to fairly rudimentary and universal tasks. In

Table 5 Questionnaire Feedback Comments. Summary of the most frequent comments. Comments were generalized to give an overall impression of how participants responded. Categories represent topical prompts to which participants responded

\begin{tabular}{lll}
\hline Category & Generalized Comment \\
\hline Criticism of the tasks & Difficulty with joystick precision and accuracy & Count \\
& Lacking of vision due to fixed camera & 5 \\
Reactions to the reaching controller & Elevator was too small & 3 \\
& Enjoyed using the reaching controller & Noted 'glitches' during reaching tasks \\
Suggestions & 'It adds realism to the game' & 3 \\
& Difficulty due to lack of depth perception & 3 \\
& Changes to the task that would add complexity & 2 \\
5 & Changes to the task that would lower complexity & 5 \\
\hline
\end{tabular}


context of the participants' SOP, their feedback regarding realism of task presentation reflects positively on their SOP. However, it seems the overall difficulty of performing the tasks in VR was likely a key factor in the lack of change in the IPQ realism score.

Participants also largely viewed the simulator as useful for learning to use a PW. It may have been some time since experienced users first learned to use their PW and may have forgotten what the specific challenges they had to go through. However, expert users will have likely experienced a wider range of situations and challenges, compared to beginners and therefore would have a more complete perspective on the learning utility of the simulator.

\section{Task performance}

We compared task performance between RW and VR environments. We found that, in two of three tasks (Desk and Elevator), task completion time, time spent reaching, number of joystick movements, and number of reaching movements were all significantly greater when the task was performed in VR compared to RW. Only the Door task showed any concordant performance measures.

Joystick movements were represented by continuous deviation from the joystick neutral position and therefore reflect distinct PW manoeuvres. As such, concordant joystick movements in the Door task indicate that participants followed similar manoeuvring sequences in VR and PW; discordant joystick movements for the Desk and Elevator tasks indicate that participants navigated to objectives differently.

Few studies have directly compared PW task performance between VR and RW. Similar to our results, the study by Harrison and colleagues [22] reported greater task completion times and a greater number of discrete manoeuvres in VR compared to RW when comparing several manoeuvring tasks and one route-finding task. However, the previous study involving the miWe simulator [20] showed no significant difference in task completion times in 4/7 Wheelchair Skills Test [23] type tasks and no significant difference in the number of joystick movements in $6 / 7$ of these tasks. The authors noted that the tasks with significantly greater times and movements were the most difficult ones. Specifically, in their 'Door (Push)' task, participants lined up with the door, 'pushed' the door open, drove through, turned around, and closed the door. In comparison, our Door task required no turning and minimal manoeuvring through tight spaces. Therefore, as our simplest task, it is unsurprising that the Door task was the one that did show concordant performance in total task time and joystick control.

Our video task analysis showed a higher risk of collisions in VR compared to RW. Of note, collisions occurred in $100 \%$ of Desk trials and $93 \%$ of Elevator trials in VR. Similarly, Harrison and colleagues [22] counted a total of 4 collisions over all their RW trials and 140 over all VR trials, in their manoeuvring and route-finding tasks. The authors noted that collision rates were greatest in tasks that required turning. Furthermore, Archambault and colleagues [20] noted frequent collisions and adjustments in VR compared to RW for their most complex manoeuvring tasks. This is consistent with our results where the greatest relative risk of collisions in VR were with regard to the Desk and Elevator tasks, which required turning in tight spaces. Therefore, corrective and repeat movements for collisions likely contributed to greater task times. The poor agreement of the driving behaviours 'advance straight' (Door task) and 'fluid driveto-park' further suggests that navigation was overall more efficient in RW than in VR.

The poorer navigation performance is reflective of the participants' feedback regarding difficulty of control and may be explained by a number of simulator limitations. The miWe simulator has a fixed field of view (FOV) of the virtual world; the virtual camera is fixed in the forward position and participants cannot easily 'look around' their wheelchair to focus on obstacles. Moreover, the miWe system uses a 2D, monoscopic display instead of a 3D stereoscopic display, meaning participants lacked depth perception for judging distances to obstacles. Participants also responded on the questionnaire that the joystick control of the PW was unrealistic, e.g. wide turn radius and needing improvement in precision and accuracy. Together, these limitations make obstacle avoidance, especially in tight spaces, more challenging in VR than in RW and likely make a major contribution to the observed discordance in navigation performance.

For cognitive skills such as path finding and overall task planning, however, participants tended to adopt concordant (moderate to excellent agreement) strategies when making navigation choices in VR and RW: they generally parked in the same position relative to the reaching target and they approached the buttons on the elevator in the same relative orientation. Also, the majority of observed reaching behaviours and strategies were concordant (moderate to excellent agreement) for each task in VR and RW: participants tended to use the same hand for reaching a given object and had similar start-of-reach timing relative to parking. This suggests that, while participants may have driven less efficiently in VR compared to RW, they still performed both route finding and task planning (for navigation and reaching components) in similar ways.

Reaching movements were represented by distinct arm movements to a target. Similar to the joystick results, participants showed concordant reaching sequences in the Door task and discordant reaching sequences in the Desk and Elevator tasks. 
To our knowledge, this study is the first to compare reaching performance between VR and RW in context of using a PW. However, there is much research on motor performance in VR with respect to upper-limb rehabilitation [9]. Of note, Viau and colleagues [24] compared motor performance and movement patterns between VR (2D display) and RW during a reach-grasprelease task. These authors reported similar overall movement strategies between the two environments, but different movement with respect to degree of elbow and wrist extension. However, other studies have demonstrated mixed results [12, 25-27]. Some of the differences in performance in VR (both 2D and 3D) have been attributed to the presence or absence of haptic feedback [28] and display platforms [29].

The lack of haptic feedback and depth perception in our study likely contributed to the greater difficulty reported by participants and explain the greater number of reaching movements (Door and Elevator tasks) and reaching time (all tasks) observed in our study; one participant commented in the questionnaire that they had difficulty knowing if they had pressed the button in the Elevator task, even with the visual feedback (button lighting up). Additionally, the reaching tasks in our study differed from other studies in that the starting position of the user relative to the reaching objective was variable and dependent on the participant's judgement; they decided where to park. As such, participants were required to accurately judge that the reaching objective was indeed within reach. Our results showed that, for all tasks, participants were more likely to misjudge this reaching distance in VR, i.e. higher relative risk of reaching errors.

Overall, the above mentioned limitations in the simulator may have contributed negatively to participants' SOP and likely countered potential gains in the realism, spatial presence, and general sense of presence categories of the IPQ due to our implementation of the Hydra controller and combined navigation-reaching tasks. Unfortunately, it seems the greater complexity and number of features we have for a simulator, the more opportunities there are for participants to experience unrealism in some aspect of the simulator.

A potential solution for many of these issues may be the utilization of low cost consumer-level HMDs, e.g. the Oculus Rift (Oculus VR, Irvine, USA), featuring a $1080 \mathrm{p}$ resolution stereoscopic display, a physical $90^{\circ}$ by $110^{\circ} \mathrm{FOV}$, and head motion tracking capabilities. This would provide greater visual immersion, depth perception, and enable users to look around the virtual environment using head movements and may be implemented in future versions of the simulator.

Furthermore, the upcoming Sixense STEM system (Sixense, USA) is the next generation of the Hydra motion controller. The STEM system features small blocks that feature just the motion sensor and additional haptic feedback and could be readily implemented in the miWe. These blocks could more easily be attached to a user's hands and the haptic feedback would increase the interactivity of reaching in the miWe.

\section{Conclusions}

In summary, this study on the Razer Hydra 3D motion controller provides evidence supporting its use as an interface for combined navigation-reaching tasks in the miWe simulator. SOP was increased in the involvement domain compared to the simulator without reaching; participants demonstrated concordant task behaviours and strategies and concordant kinematic performance on the least difficult task between VR and RW; and feedback from participants indicated that the combined navigation-reaching tasks were appropriate, useful for PW training, and engaging. Therefore, this device is a valid interface for training and familiarizing combined manual-navigation tasks from a task planning/strategy standpoint and may be utilized in future versions of the simulator. However, important limitations of the simulator explain discordant measures and ultimately the lackluster increases in participants' SOP; therefore, future development of the simulator must address these issues through improved hardware and refinement of the virtual environment.

We believe that, in more complex tasks, fundamental differences between VR (e.g. PW steering characteristics, depth perception, multiple interfaces) and RW are more likely to be compounded, resulting in divergent task performances. However, it is this very capacity for complex tasks that marks a key advantage of VR simulators. It allows us to expand traditional training to include, in VR, a greater variety of stakeholder-relevant tasks, scenarios, and difficulty levels. In context of this study, we may consider any situation, at home or in the community, in which PW users would need to interact using their hands, i.e. capitalizing on the integration of a 3D had motion controller. Therefore, future development will need focus on minimizing the experiential differences, with an emphasis on difficulty optimization, from the real world. Some of these developments in the miWe are underway and reported by Archambault and colleagues [30].

\section{Abbreviations \\ FOV: Field of View; IPQ: iGroup Presence Questionnaire; IQR: Interquartile Range; miWe: McGill Immersive Wheelchair Simulator; PW: Powered \\ Wheelchair; RR: Relative Risk; SOP: Sense of Presence; VR: Virtual Reality.}

\section{Competing interests}

No competing interests declared.

\section{Author's contributions}

GT carried out or was involved in every aspect of this project, including study design, integration of the motion controller into the simulator, design of the virtual environment, preparation of equipment and data collection 
space, recruitment, data collection, data processing and analysis, and manuscript preparation. PA acted in a supervisory role, providing critical feedback, assistance, and guidance at each stage of the project. He also reviewed, revised, and edited the manuscript. All authors read and approved the final manuscript.

\section{Acknowledgements}

The authors would like to thank all the participants who took part in this study. Helen Zhu and Elise Busilacchi assisted in data collection and processing. Christian Beaudoin, Frederic Bobohn, and Frederic Vicaire contributed to software development and Igor Sorokin constructed the props used in the experiment.

Gordon Tao was the recipient of a scholarship from the School of Physical and Occupational Therapy of McGill University. This project was funded in part by Le Projet Stratégique Innovant of CRIR.

Received: 21 August 2015 Accepted: 8 January 2016

Published online: 19 January 2016

\section{References}

1. Holliday P, Mihailidis A, Rolfson R, Fernie G. Understanding and measuring powered wheelchair mobility and manoeuvrability. Part I. Reach in confined spaces. Disabil Rehabil. 2005;27(16):939-49.

2. Ward AL, Sanjak M, Duffy K, Bravver E, Williams N, Nichols M, et al. Power wheelchair prescription, utilization, satisfaction, and cost for patients with amyotrophic lateral sclerosis: preliminary data for evidence-based guidelines. Arch Phys Med Rehabil. 2010;91(2):268-72.

3. Sonenblum SE, Sprigle S, Harris FH, Maurer CL. Characterization of power wheelchair use in the home and community. Arch Phys Med Rehabil. 2008:89(3):486-91.

4. Kaye HS, Kang T, LaPlante MP. Mobility device use in the United States: National Institute on Disability and Rehabilitation Research, US Department of Education. Institute for Health and Aging, University of California. 2000.

5. Kirby RL, Keeler L, Wang S, Thompson K, Theriault C. Proportion of Wheelchair Users Who Receive Wheelchair Skills Training During an Admission to a Canadian Rehabilitation Center. Top Geriatr Rehabil. 2015;31(1):58-66.

6. Linden MA, Whyatt C, Craig C, Kerr C. Efficacy of a powered wheelchair simulator for school aged children: A randomized controlled trial. Rehabil Psychol. 2013;58(4):405.

7. Archambault PS, Chong JNF, Sorrento G, Routhier F, Boissy P, editors. Comparison of powered wheelchair driving performance in a real and in a simulated environment. Virtual Rehabilitation (ICVR), 2011 International Conference on; 2011 27-29 June 2011.

8. Abellard P, Randria I, Abellard A, Ben Khelifa M, Ramanantsizehena P. Electric Wheelchair Navigation Simulators: why, when, how? In: Di Paola A, Cicirelli G, editors. Mechatronic Systems Appications. InTech; 2010.

9. Levin MF, Deutsch JE, Kafri M, Liebermann DG. Validity of Virtual Reality Environments for Sensorimotor Rehabilitation. Virtual Reality for Physical and Motor Rehabilitation. Springer: New York; 2014. p. 95-118.

10. Holden MK. Virtual environments for motor rehabilitation: review. Cyberpsychol Behav. 2005;8(3):187-211.

11. Mantovani F, Castelnuovo G. The Sense of Presence in Virtual Training: Enhancing Skills Acquisition and Transfer of Knowledge through Learning Experience in Virtual Environments. In: Riva G., D.F. editor. Being There: Concepts, Effects and Measurement of User Presence in Synthetic Environments. Amsterdam. IOS Press. 2003:167-82.

12. Ma H-I, Hwang W-J, Wang C-Y, Fang J-J, Leong I-F, Wang T-Y. Trunk-arm coordination in reaching for moving targets in people with Parkinson's disease: Comparison between virtual and physical reality. Hum Mov Sci. 2012;31(5):1340-52.

13. Zhou $\mathrm{H}, \mathrm{Hu} \mathrm{H}$. Human motion tracking for rehabilitation-A survey. Biomed Signal Process Control. 2008;3(1):1-18. doi:10.1016/j.bspc.2007.09.001.

14. Subramanian S, Knaut LA, Beaudoin C, McFadyen BJ, Feldman AG, Levin MF. Virtual reality environments for post-stroke arm rehabilitation. J Neuroeng Rehabil. 2007:4:20. doi:10.1186/1743-0003-4-20.

15. IJsselsteijn W. Elements of a multi-level theory of presence: Phenomenology, mental processing and neural correlates. Proc PRESENCE. 2002;2002:245-59.

16. Seegert A. Doing there vs. being there: performing presence in interactive fiction. J Gaming Virtual Worlds. 2009;1(1):23-37.
17. Nasreddine ZS, Phillips NA, Bédirian V, Charbonneau S, Whitehead V, Collin I, et al. The Montreal Cognitive Assessment, MoCA: a brief screening tool for mild cognitive impairment. J Am Geriatr Soc. 2005;53(4):695-9.

18. Nichols S, Haldane C, Wilson JR. Measurement of presence and its consequences in virtual environments. Int J Hum-Comput St. 2000;52(3):471-91. doi:10.1006/ijhc.1999.0343.

19. Schubert T, Friedmann F, Regenbrecht $H$. The experience of presence: Factor analytic insights. Presence. 2001;10(3):266-81.

20. Archambault PS, Tremblay S, Cachecho S, Routhier F, Boissy P. Driving performance in a power wheelchair simulator. Disabil Rehabil Assist Technol. 2012;7(3):226-33. doi:10.3109/17483107.2011.625072.

21. Fleiss J. Statistical methods tor rates and proportions. Nueva York: Wiley; 1981. p. 8.

22. Harrison A, Derwent G, Enticknap A, Rose F, Attree E. Application of virtual reality technology to the assessment and training of powered wheelchair users. ICDVRAT 2000. 2000

23. University D. Wheelchair Skills Test (WST) Power WC - Wheelchair User, Version 4.2.2. Dalhousie University. 2013. http://www. wheelchairskillsprogram.ca/eng/. Accessed 01/08/2014 2014.

24. Viau A, Feldman AG, McFadyen BJ, Levin MF. Reaching in reality and virtual reality: a comparison of movement kinematics in healthy subjects and in adults with hemiparesis. J Neuroeng Rehabil. 2004;1(1):11. doi:10.1186/17430003-1-11.

25. Liebermann DG, Berman S, Weiss PL, Levin MF. Kinematics of reaching movements in a 2-D virtual environment in adults with and without stroke. IEEE Trans Neural Syst Rehabil Eng. 2012;20(6):778-87.

26. Wang C-Y, Hwang W-J, Fang J-J, Sheu C-F, Leong I-F, Ma H-I. Comparison of virtual reality versus physical reality on movement characteristics of persons with Parkinson's disease: effects of moving targets. Arch Phys Med Rehabil. 2011;92(8):1238-45.

27. Lott A, Bisson E, Lajoie Y, McComas J, Sveistrup H. The effect of two types of virtual reality on voluntary center of pressure displacement. Cyberpsychol Behav. 2003;6(5):477-85.

28. Magdalon EC, Michaelsen SM, Quevedo AA, Levin MF. Comparison of grasping movements made by healthy subjects in a 3-dimensional immersive virtual versus physical environment. Acta Psychol (Amst). 2011;138(1):126-34

29. Subramanian SK, Levin MF. Viewing medium affects arm motor performance in 3D virtual environments. J Neuroeng Rehabil. 2011:8:36.

30. Archambault PS, Norouzi NG, Kairy D, Solomon JM, Levin MF. Towards Establishing Clinical Guidelines for an Arm Rehabilitation Virtual Reality System. Replace, Repair, Restore, Relieve-Bridging Clinical and Engineering Solutions in Neurorehabilitation. Springer: New York; 2014. p. 263-70.

\section{Submit your next manuscript to BioMed Central and we will help you at every step:}

- We accept pre-submission inquiries

- Our selector tool helps you to find the most relevant journal

- We provide round the clock customer support

- Convenient online submission

- Thorough peer review

- Inclusion in PubMed and all major indexing services

- Maximum visibility for your research

Submit your manuscript at www.biomedcentral.com/submit 